\title{
Differential Geometry of Bipartite Quantum States
}

\author{
Zuhuan $\mathrm{Yu}^{a *}$, Xianqing Jost-Li ${ }^{b \dagger}$, Qingzhong $\mathrm{Li}^{a \ddagger}$, Jintao Lva ${ }^{a \S}$, and Shao-Ming Fei ${ }^{a, b \uparrow}$ \\ a Department of Mathematics, Capital Normal University, Beijing 10003r, China \\ ${ }^{b}$ Max Planck Institute for Mathematics in the Sciences, D-04103 Leipzig, Germany
}

\begin{abstract}
We investigate the differential geometry of bipartite quantum states. In particular the manifold structures of pure bipartite states are studied in detail. The manifolds with respect to all normalized pure states of arbitrarily given Schmidt ranks or Schmidt coefficients are explicitly presented. The dimensions of the related manifolds are calculated.
\end{abstract}

PACS numbers: 03.67.-a, 03.65.Ud, 02.40.-k

Keywords: Differential geometry, Bipartite states, Manifold

\footnotetext{
* e-mail: yuzh@mail.cnu.edu.cn

$\dagger$ e-mail: Xianqing.Li-Jost@mis.mpg.de

$\ddagger$ e-mail: liqzh@mail.cnu.edu.cn

$\S$ e-mail: lvjintao@sohu.com

ฯ e-mail: fei@wiener.iam.uni-bonn.de
} 
Quantum entanglement constitutes the most important resource in quantum information processing such as quantum teleportation, dense coding, quantum cryptography, quantum error correction and quantum repeater [1]. The marvelous properties of quantum entanglement are from the special structures of the multipartite quantum states. Great efforts have been focused on the proper description and quantification of quantum entanglement [2], the separability [3], the equivalence of quantum states under local unitary transformations or under stochastic local operations and classical communication (SLOCC) for multipartite quantum systems [4]].

The geometry of quantum states on a single vector space has been discussed in [5, 6] recently. Let $\mathcal{H}$ be an $n$-dimensional complex Hilbert space. The space of density matrices on $\mathcal{H}, D(\mathcal{H})$, is naturally a manifold stratified space with the stratification induced by the rank of the state. The space of all density matrices with rank $r, D^{r}(\mathcal{H}), r=1,2, \cdots, n$, is a smooth and connected manifold of real dimension $2 n r-r^{2}-1$. In particular, $D^{1}(\mathcal{H})$ is the set of pure states. Every element of $D(\mathcal{H})$ is a convex combination of points from $D^{1}(\mathcal{H})$. It is shown that $D^{1}(\mathcal{H})$ is a complex manifold which is isomorphic to the $n-1$ dimensional complex projective space, $D^{1}(\mathcal{H}) \simeq C P^{n-1}$, with a metric $g$ determined by the inner product $\langle M, N\rangle=\frac{1}{2} \operatorname{Tr} M N$ for density matrices $M$ and $N$. One can define the Hermitian structure $h$ on $D^{1}(\mathcal{H})$ by $g$. In fact, by straightforward calculation, we have

$$
h^{(\alpha)}=\sum_{k, j} h_{k j}^{(\alpha)} \mathrm{d} z_{k} \otimes \mathrm{d} \overline{z_{j}}, \quad h^{(\alpha)}=h_{\mid D_{\alpha}}, \quad \alpha=1, \ldots, n,
$$

where

$$
h_{k j}^{(\alpha)}=\frac{\left(1+\sum_{l=1, l \neq \alpha}^{n}\left|z_{l}\right|^{2}\right) \delta_{k j}-z_{j} \overline{z_{k}}}{\left(1+\sum_{l=1, l \neq \alpha}^{n}\left|z_{l}\right|^{2}\right)},
$$

$D_{\alpha}$ is the $\alpha$-th coordinate chart with local complex coordinates $z$ and $\bar{z}$. Hence it is clear that $h$ differs from the Fubini-Study metric on $C P^{n-1}$ by a constant multiple.

The quantum entanglement concerns composite systems. In [7] the entanglement has been discussed in the view of geometry. In this paper we investigate the manifold structures and classification of pure bipartite states. We consider quantum states on $\mathcal{H}=\mathcal{H}_{1} \otimes \mathcal{H}_{2}$, where $\mathcal{H}_{1}$ and $\mathcal{H}_{2}$ are respectively $n$ and $m(n \leq m)$ dimensional complex Hilbert spaces. We present the explicit manifold constituted by the states with certain Schmidt ranks or with given Schmidt coefficients, and calculate the dimensions of the related manifolds. 
For the convenience, in the following in stead of $|x\rangle$, we simply denote $x$ as a vector in $\mathcal{H}$ and denote $D^{1}(\mathcal{H})$ as the set of all $x \in \mathcal{H}$. For any $x \in \mathcal{H}, x$ can be written as the summation of tensor products,

$$
x=x_{1} \otimes y_{1}+x_{2} \otimes y_{2}+\cdots+x_{k} \otimes y_{k}, \quad k \in \mathbb{N},
$$

where $x_{i} \in \mathcal{H}_{1}, y_{i} \in \mathcal{H}_{2}$. We call the expression (11) linearly independent if $x_{1}, x_{2}, \cdots, x_{k}$; $y_{1}, y_{2}, \cdots, y_{k}$ are linearly independent vectors respectively. We say the length of $x$ is $k$ if (1) is a linearly independent expression. In fact one can easily prove that the length is just the Schmidt rank and the Schmidt decomposition is a special expression of a linearly independent one. Therefore the length of $x$ in all linearly independent expressions is the same and the terms of tensor products contained in the linearly independent expression of $x$ are the least in all other possible expressions of $x$.

[Lemma] If $x \in \mathcal{H}=\mathcal{H}_{1} \otimes \mathcal{H}_{2}$ has the following two linearly independent expressions

$$
x=x_{1} \otimes y_{1}+x_{2} \otimes y_{2}+\cdots+x_{k} \otimes y_{k}, x=w_{1} \otimes z_{1}+w_{2} \otimes z_{2}+\cdots+w_{k} \otimes z_{k},
$$

then there exists a non-degenerate $k \times k$ matrix $C$ such that

$$
\left(z_{1}, \cdots, z_{k}\right)=\left(y_{1}, \cdots, y_{k}\right) C, \quad\left(w_{1}, \cdots, w_{k}\right)=\left(x_{1}, \cdots, x_{k}\right)\left(C^{t}\right)^{-1}
$$

[Proof] Expanding $x_{1}, x_{2}, \cdots, x_{k}$ to be the basis $x_{1}, \cdots, x_{k}, x_{k+1}, \cdots, x_{n}$ in $\mathcal{H}_{1}$ and $y_{1}, y_{2}, \cdots, y_{k}$ to be the basis $y_{1}, \cdots, y_{k}, y_{k+1}, \cdots, y_{m}$ in $\mathcal{H}_{2}$, we have

$$
w_{j}=\sum_{i=1}^{n} a_{j i} x_{i}, z_{j}=\sum_{i=1}^{m} b_{j i} y_{i}
$$

for some $a_{j i}, b_{j i} \in \mathbb{C}$. Then from (2) we have

$$
\sum_{i=1}^{n} \sum_{s=1}^{m}\left(\sum_{j=1}^{k} a_{j i} b_{j s}\right) x_{i} \otimes y_{s}=\sum_{j=1}^{k} x_{j} \otimes y_{j} .
$$

Denote $A$ (resp. $B$ ) the matrix with entries $a_{i j}$ (resp. $\left.b_{i j}\right)$. As $\left\{x_{i} \otimes y_{s}: j=\right.$ $1,2, \cdots, n ; s=1,2, \cdots, m\}$ is a basis of $\mathcal{H}_{1} \otimes \mathcal{H}_{2}$, from (41) we have

$$
A^{t} B=\left(\begin{array}{cc}
E_{k} & 0 \\
0 & 0
\end{array}\right)_{n \times m}
$$

where $E_{k}$ is the identity matrix of order $k$. If we rewrite $A$ and $B$ as block matrices $A=\left(\begin{array}{ll}A_{k k} & A_{k, n-k}\end{array}\right), B=\left(\begin{array}{ll}B_{k k} & B_{k, m-k}\end{array}\right)$, then (5) gives rise to $A_{k k}^{t} B_{k k}=E_{k}, B_{k, m-k}=0$, 
$A_{k, n-k}=0$. Namely, $A=\left(\begin{array}{ll}A_{k k} & 0\end{array}\right), B=\left(\begin{array}{ll}B_{k k} & 0\end{array}\right)$. Set $C=A_{k k}^{-1}$, we obtain $B_{k k}=\left(A_{k k}^{t}\right)^{-1}$ and $\left(w_{1}, \cdots, w_{k}\right)=\left(x_{1}, \cdots, x_{k}\right)\left(C^{t}\right)^{-1},\left(z_{1}, \cdots, z_{k}\right)=\left(y_{1}, \cdots, y_{k}\right) B_{k k}^{t}=\left(y_{1}, \cdots, y_{k}\right) C$.

[Theorem 1] Let $D_{k}^{1}(\mathcal{H})$, a submanifold of $D^{1}(\mathcal{H})$, be the set of all normalized pure states with length $k, D_{k}^{1}(\mathcal{H})=\left\{x \in \mathcal{H}\right.$, the length of $x$ is $\left.k,\|x\|^{2}=1\right\}$. We have

$$
D_{k}^{1}(\mathcal{H}) \simeq G(n, k) \times\left(C P^{k^{2}-1} \backslash \bar{M}\right) \times G(m, k),
$$

where $\bar{M}$ is a hypersurface of $C P^{k^{2}-1}, G(n, k)$ is the Grassmannian manifold.

[Proof] We first prove that there is a one-to-one correspondence between $D_{k}^{1}(\mathcal{H})$ and $G(n, k) \times\left(C P^{k^{2}-1} \backslash \bar{M}\right) \times G(m, k)$.

For $x \in D_{k}^{1}(\mathcal{H})$, suppose $x=x_{1} \otimes y_{1}+x_{2} \otimes y_{2}+\cdots+x_{k} \otimes y_{k}$ is a linearly independent expression of $x$. Because $y_{1}, \cdots, y_{k}$ are linearly independent, $y_{1}, \cdots, y_{k}$ span a $k$-dimensional subspace $D_{k}$ of $\mathcal{H}_{2}$. We fix an orthonormal basis $y_{1}^{0}, \cdots, y_{k}^{0}$ in $D_{k}$ and assume $\left(y_{1}^{0} \cdots y_{k}^{0}\right)=\left(y_{1} \cdots y_{k}\right) A$, where $A$ is a non-degenerate complex $k \times k$ matrix. If we keep $x$ unchanged, from Lemma $x_{1}, \cdots, x_{k}$ are transformed correspondingly to $x_{1}^{\prime}, \cdots, x_{k}^{\prime}$, $\left(x_{1}^{\prime} \cdots x_{k}^{\prime}\right)=\left(x_{1} \cdots x_{k}\right)\left(A^{t}\right)^{-1}$.

A $k$-dimensional subspace of $\mathcal{H}_{2}$ just corresponds to a point in a Grassmannian manifold $G(m, k)$. As $x_{1}^{\prime}, \cdots, x_{k}^{\prime}$ in the expression $x=x_{1}^{\prime} \otimes y_{1}^{0}+\cdots+x_{k}^{\prime} \otimes y_{k}^{0}$ are linearly independent, they span a $k$-dimensional subspace $C_{k}$ of $\mathcal{H}_{1}$. If we fix an orthonormal basis $x_{1}^{0}, \cdots, x_{k}^{0}$ in $C_{k}$, then there exists a unique non-degenerate $k \times k$ matrix $G$ such that $\left(x_{1}, \cdots, x_{k}\right)=\left(x_{1}^{0}, \cdots, x_{k}^{0}\right) G$. A $k$-dimensional subspace of $\mathcal{H}_{1}$ just corresponds to a point in a Grassmannian manifold $G(n, k)$. Suppose $\left(x_{1}^{\prime}, \cdots, x_{k}^{\prime}\right)=\left(x_{1}^{0}, \cdots, x_{k}^{0}\right) B$, where $B$ is a $k \times k$ complex matrix with entries $b_{i j}$ satisfying $\sum_{i, j=1}^{k}\left|b_{i j}\right|^{2}=1, \operatorname{det}(B) \neq 0$. Then all $B=\left(b_{i j}\right)_{i, j=1}^{k}$ constitute a set $D$ which can be viewed as a subset of the identity ball $S^{k^{2}-1}$ in $\mathbf{C}^{k^{2}}$, where

$$
S^{k^{2}-1}=\left\{\left(b_{11}, b_{21}, \cdots, b_{k 1}, b_{12}, \cdots, b_{k 2}, \cdots, b_{k k}\right): \sum_{i, j=1}^{k}\left|b_{i j}\right|^{2}=1, b_{i j} \in \mathbb{C}\right\} .
$$

Moreover, $D$ is an open subset in $S^{k^{2}-1}$.

In summary, to determine $x_{1}^{\prime}, \cdots, x_{k}^{\prime}$, we need to determine the $k$-dimensional subspace $C_{k}$ which is spanned by $x_{1}^{\prime}, \cdots, x_{k}^{\prime}$ and the nondegenerate $k \times k$ matrix $B$ associated with $x_{1}^{\prime}, \cdots, x_{k}^{\prime}$, i.e. a point of Grassmannian manifold $G(n, k)$ and a point of $D$ are determined.

We define $A \sim B$ iff there exists $\theta \in \mathbb{R}$ such that $A=e^{i \theta} B$ and denote the equivalence class containing $A$ by $[A]$, then

$$
S^{k^{2}-1} / \sim=C P^{k^{2}-1}
$$


Define

$$
\begin{aligned}
\pi: S^{k^{2}-1} & \longrightarrow C P^{k^{2}-1} \\
A & \longrightarrow[A] .
\end{aligned}
$$

Then $\pi$ is an open map. Suppose the image of $D$ under $\pi$ is $\bar{D}$ which is an open subset of $C P^{k^{2}-1}$, so it is an open submanifold. Suppose $\bar{M}=C P^{k^{2}-1} \backslash \bar{D}$, i.e. $\bar{M}$ is the image of the set under the map $\pi$ which consists of the points contained in $S^{k^{2}-1} \operatorname{satisfying} \operatorname{det}(B)=0$ and $\bar{M}$ is a hypersurface of $C P^{k^{2}-1}$. So we have

$$
\bar{D}=C P^{k^{2}-1} \backslash \bar{M}
$$

As $\left(e^{i \theta} x_{1}^{\prime}, \cdots, e^{i \theta} x_{k}^{\prime}\right)=e^{i \theta}\left(x_{1}^{0}, \cdots, x_{k}^{0}\right) B=\left(x_{1}^{0}, \cdots, x_{k}^{0}\right)\left(e^{i \theta} B\right)$, the action of $e^{i \theta}$ on $x$ can be viewed as on matrix $B$ associated with $x_{1}^{\prime}, \cdots, x_{k}^{\prime}$. So the equivalence class $[x]$ containing $x$ corresponds to the equivalence class $[B]$ which contains $B$, i.e. $[x]$ corresponds a point in $\bar{D}$. Hence, a pure state $x$ corresponds to a unique point $p$ in $G(n, k) \times\left(C P^{k^{2}-1} \backslash \bar{M}\right) \times G(m, k)$, where the coordinates of $p$ are determined uniquely by the $k$-dimensional subspace $D_{k}$ in $\mathcal{H}_{2}$ spanned by $y_{1}, \cdots, y_{k}$, the $k$-dimensional subspace $C_{k}$ in $\mathcal{H}_{1}$ spanned by $x_{1}, \cdots, x_{k}$ and $[B]$. We denote this kind of correspondence as $F$. One can easily prove that $F$ is surjective and injective. So we get a one-to-one correspondence between $D_{k}^{1}(\mathcal{H})$ and $G(n, k) \times$ $\left(C P^{k^{2}-1} \backslash \bar{M}\right) \times G(m, k)$. Moreover from the above proof we know that $F$ is smooth.

We now imbed $G(n, k) \times\left(C P^{k^{2}-1} \backslash \bar{M}\right) \times G(m, k)$ to $C P^{m n-1}$ according to $F$. For arbitrary $p \in G(n, k) \times\left(C P^{k^{2}-1} \backslash \bar{M}\right) \times G(m, k)$, the coordinates of $p$ have the form,

$$
\left(x_{1, k+1}, \cdots, x_{2 n}, \cdots, x_{k n}, a_{12}, \cdots, a_{k k}, y_{1, k+1}, \cdots, y_{k m}\right) .
$$

Let us write the coordinates $\left(x_{1, k+1}, x_{1, k+2}, \cdots, x_{k n}\right)$ in $G(n, k)$ in the matrix form

$$
X=\left(\begin{array}{ccccccc}
1 & 0 & \cdots & 0 & x_{1, k+1} & \cdots & x_{1 n} \\
0 & 1 & \cdots & 0 & x_{2, k+1} & \cdots & x_{2 n} \\
\cdots & \cdots & \cdots & \cdots & \cdots & \cdots & \cdots \\
0 & 0 & \cdots & 1 & x_{k, k+1} & \cdots & x_{k n}
\end{array}\right) \text {, }
$$

and the coordinates $\left(a_{12}, \cdots, a_{1 k}, \cdots, a_{k k}\right)$ in $C P^{k^{2}-1}$ in the form

$$
A=\left(\begin{array}{cccc}
1 & a_{12} & \cdots & a_{1 k} \\
a_{21} & a_{22} & \cdots & a_{2 k} \\
\cdots & \cdots & \cdots & \cdots \\
a_{k 1} & a_{k 2} & \cdots & a_{k k}
\end{array}\right)
$$


Set $X^{t} A=B$. Then $B=\left(b_{i j}\right)$ is an $n \times k$ matrix. Let $e_{1}, \cdots, e_{n}\left(\right.$ resp. $\left.d_{1}, \cdots, d_{m}\right)$ be an orthonormal basis in $\mathcal{H}_{1}\left(\right.$ resp. $\left.\mathcal{H}_{2}\right)$. Take

$$
x_{1}=\sum_{j=1}^{n} b_{j 1} e_{j}, x_{2}=\sum_{j=1}^{n} b_{j 2} e_{j}, \cdots, x_{k}=\sum_{j=1}^{n} b_{j k} e_{j}
$$

and

$$
y_{1}=\sum_{j=1}^{m} y_{1 j} d_{j}, y_{2}=\sum_{j=1}^{m} y_{2 j} d_{j}, \cdots, y_{k}=\sum_{j=1}^{m} y_{k j} d_{j},
$$

where $y_{i j}$ are the entries of the matrix $Y$,

$$
Y=\left(\begin{array}{ccccccc}
1 & 0 & \cdots & 0 & y_{1, k+1} & \cdots & y_{1 m} \\
0 & 1 & \cdots & 0 & y_{2, k+1} & \cdots & y_{2 m} \\
\cdots & \cdots & \cdots & \cdots & \cdots & \cdots & \cdots \\
0 & 0 & \cdots & 1 & y_{k, k+1} & \cdots & y_{k m}
\end{array}\right)
$$

then both $x_{1}, \cdots, x_{k}$ and $y_{1}, \cdots, y_{k}$ are linearly independent respectively.

Let

$$
x=x_{1} \otimes y_{1}+\cdots+x_{k} \otimes y_{k}=\sum_{j=1}^{n} \sum_{s=1}^{m}\left(\sum_{l=1}^{k} b_{j l} y_{l s}\right) e_{j} \otimes d_{s} .
$$

Then $x \in D_{k}^{1}(\mathcal{H})$ is the image of $p$ under $F$. Since $\mathcal{H}_{1} \simeq \mathbb{C}^{n}, \mathcal{H}_{2} \simeq \mathbb{C}^{m}$ and $D^{1}(\mathcal{H}) \simeq C P^{n m-1}$, we can define the imbedding:

$$
\begin{array}{cl}
f: G(n, k) \times\left(C P^{k^{2}-1} \backslash \bar{M}\right) \times G(m, k) & \longrightarrow C P^{m n-1} \\
p & \longrightarrow q
\end{array}
$$

where $q=f(p)=x$. The homogeneous coordinates of $q$ are given by $q=$ $\left(d_{11}, d_{12}, \cdots, d_{1 m}, d_{21}, \cdots, d_{2 m}, \cdots, d_{n m}\right)$, where $d_{j s}=\sum_{l=1}^{k} b_{j l} y_{l s}=\sum_{l=1}^{k} \sum_{t=1}^{k} x_{t j} a_{t l} y_{l s}(j=$ $1, \cdots, n, s=1, \cdots, m)$. Then the coordinate components of $q$ are polynomial of the coordinate components of $p$. Hence, the imbedding $f$ is non-degenerate holomorphic mapping. Moreover, we have

$$
f\left(G(n, k) \times\left(C P^{k^{2}-1} \backslash \bar{M}\right) \times G(m, k)\right)=D_{k}^{1}(\mathcal{H}) .
$$

Therefore $D_{k}^{1}(\mathcal{H})$ is a complex submanifold of $C P^{m n-1}$ ( i.e. $D^{1}(\mathcal{H})$ ), and

$$
D_{k}^{1}(\mathcal{H}) \simeq G(n, k) \times\left(C P^{k^{2}-1} \backslash \bar{M}\right) \times G(m, k) .
$$


Theorem 2 The subset $D_{k}^{1}\left(\mu_{1}, \cdots, \mu_{k}\right)$ of $D_{k}^{1}(\mathcal{H})$ of pure states with the Schmidt coefficients $\mu_{1} \geqslant \mu_{2} \geqslant \cdots \geqslant \mu_{k}$ is a submanifold of real dimension $2 k(m+n-k)-k-1$, which is diffeomorphically equivalent to a manifold

$$
\left(C P^{n-1} \times C P^{m-1}\right) \times \cdots \times\left(C P^{n-k} \times C P^{m-k}\right) \times T^{k-1},
$$

where $T^{k-1}$ is a torus of real dimension $k-1$.

[proof] For any pure state $[e]$ of $D_{k}^{1}(\mathcal{H})$, the unit vector $e$ has the following Schmidt representation $e=\mu_{1} a_{1} \otimes b_{1}+\cdots+\mu_{k} a_{k} \otimes b_{k}$, where $a_{i}^{s} s$ and $b_{i}^{s} s$ are orthonormal vectors in $\mathcal{H}_{1}$ and $\mathcal{H}_{2}$ respectively, and $\mu_{i}^{\prime} s$ are Schmidt coefficients of $e$, we assume that $\mu_{1} \geqslant$ $\mu_{2} \geqslant \cdots \geqslant \mu_{k}$. Consider the element $\tilde{e}$ which has the same Schmidt coefficients as $e$, $\tilde{e}=\mu_{1} \tilde{a_{1}} \otimes \tilde{b_{1}}+\cdots+\mu_{k} \tilde{a_{k}} \otimes \tilde{b_{k}}$, and $\left[a_{i}\right]=\left[\tilde{a_{i}}\right] \in D^{1}\left(\mathcal{H}_{1}\right), \quad\left[b_{i}\right]=\left[\tilde{b_{i}}\right] \in D^{1}\left(\mathcal{H}_{2}\right), \quad i=1, \cdots, k$. Hence $\tilde{e}$ must have the form $\tilde{e}=\mu_{1} e^{i \theta_{1}} a_{1} \otimes b_{1}+\cdots+\mu_{k} e^{i \theta_{k}} a_{k} \otimes b_{k}$, and $[\tilde{e}]$ constitute a set

$$
\left\{\left(\left[a_{1}\right],\left[b_{1}\right], \cdots,\left[a_{k}\right],\left[b_{k}\right], e^{i \beta_{1}}, \cdots, e^{i \beta_{k-1}}\right) \mid \beta_{1}, \cdots, \beta_{k-1} \in \mathbb{R}\right\} \simeq T^{k-1} .
$$

Then all the pure states with the same Schmidt coefficients $\mu_{1} \geqslant \mu_{2} \geqslant \cdots \geqslant \mu_{k}$ constitute a set which is equivalent to a manifold $\left(C P^{n-1} \times C P^{m-1}\right) \times \cdots \times\left(C P^{n-k} \times C P^{m-k}\right) \times T^{k-1}$, which is of real dimension $2 k(m+n-k)-k-1$.

As a simple example, let us first take $\operatorname{dim}\left(\mathcal{H}_{1}\right)=\operatorname{dim}\left(\mathcal{H}_{2}\right)=3, k=1$. For arbitrary $x \in D^{1}\left(\mathcal{H}_{1}\right), y \in D^{1}\left(\mathcal{H}_{2}\right)$, by the Segre imbedding we have $\operatorname{Seg}(x, y)=|x \otimes y\rangle\langle x \otimes y|$. As $w=x \otimes y \in D_{1}^{1}(\mathcal{H})$, one gets

$$
\operatorname{Seg}\left(D^{1}\left(\mathcal{H}_{1}\right) \times D^{1}\left(\mathcal{H}_{2}\right)\right) \subset D_{1}^{1}(\mathcal{H})
$$

And for arbitrary $w \in D_{1}^{1}(\mathcal{H})$, there exist $x \in \mathcal{H}_{1}, y \in \mathcal{H}_{2}$ such that $w=x \otimes y=\operatorname{Seg}(x, y)$. The Segre imbedding Seg : $D^{1}\left(\mathcal{H}_{1}\right) \times D^{1}\left(\mathcal{H}_{2}\right) \rightarrow D_{1}^{1}(\mathcal{H})$ is a surjective map to $D_{1}^{1}(\mathcal{H})$. Hence, we have $\operatorname{Seg}\left(D^{1}\left(\mathcal{H}_{1}\right) \times D^{1}\left(\mathcal{H}_{2}\right)\right)=D_{1}^{1}(\mathcal{H})$. Therefore $D_{1}^{1}(\mathcal{H}) \cong D^{1}\left(\mathcal{H}_{1}\right) \times D^{1}\left(\mathcal{H}_{2}\right) \simeq$ $C P^{2} \times C P^{2}$. From Theorem 1, in this case $k^{2}-1=0$. We get $D_{1}^{1}(\mathcal{H}) \simeq C P^{2} \times C P^{2}$.

As a more complicated case, we consider $\operatorname{dim}\left(\mathcal{H}_{1}\right)=3, \operatorname{dim}\left(\mathcal{H}_{2}\right)=4$, and $k=2$. For arbitrary $p \in G(3,2) \times\left(C P^{3} \backslash \bar{M}\right) \times G(4,2)$ with coordinate $p=\left(x_{13}, x_{23}, a_{12}, a_{21}, a_{22}, y_{13}\right.$, $\left.y_{14}, y_{23}, y_{24}\right)$, set

$$
X=\left(\begin{array}{lll}
1 & 0 & x_{13} \\
0 & 1 & x_{23}
\end{array}\right), \quad A=\left(\begin{array}{cc}
1 & a_{12} \\
a_{21} & a_{22}
\end{array}\right), \quad Y=\left(\begin{array}{llll}
1 & 0 & y_{13} & y_{14} \\
0 & 1 & y_{23} & y_{24}
\end{array}\right)
$$


Then

$$
X^{t} A=\left(\begin{array}{cc}
1 & a_{12} \\
a_{21} & a_{22} \\
x_{13}+a_{21} x_{23} & a_{12} x_{13}+x_{23} a_{22}
\end{array}\right) \text {. }
$$

We take $x_{1}=e_{1}+a_{21} e_{2}+\left(x_{13}+a_{21} x_{23}\right) e_{3}, x_{2}=a_{12} e_{1}+a_{22} e_{2}+\left(a_{12} x_{13}+x_{23} a_{22}\right) e_{3}, y_{1}=$ $d_{1}+y_{13} d_{3}+y_{14} d_{4}, y_{2}=d_{2}+y_{23} d_{3}+y_{24} d_{4}$. Let $x=x_{1} \otimes y_{1}+x_{2} \otimes y_{2}$. Since $x_{1}, x_{2} ; y_{1}, y_{2}$ are linearly independent respectively, we have $x \in D_{2}^{1}(\mathcal{H})$.

For arbitrary $x \in D_{2}^{1}(\mathcal{H})$, suppose $x=x_{1} \otimes y_{1}+x_{2} \otimes y_{2}$, then $y_{1}, y_{2}$ span a unique 2-dimensional subspace $D_{2}$ of $\mathcal{H}_{2}$. We fix an orthonormal basis $y_{1}^{0}, y_{2}^{0}$ in $D_{2}$ and suppose $\left(y_{1}^{0}, y_{2}^{0}\right)=\left(y_{1}, y_{2}\right) A$, where $A$ is a non-degenerate complex $2 \times 2$ matrix. At the same time, suppose that $x_{1}, x_{2}$ are transformed correspondingly to $x_{1}^{\prime}, x_{2}^{\prime},\left(x_{1}^{\prime}, x_{2}^{\prime}\right)=\left(x_{1}, x_{2}\right)\left(A^{t}\right)^{-1}$. Then $x=x_{1}^{\prime} \otimes y_{1}^{0}+x_{2}^{\prime} \otimes y_{2}^{0}$, and $x_{1}^{\prime}, x_{2}^{\prime}$ generate a unique 2-dimensional subspace $C_{2}$ of $\mathcal{H}_{1}$. We fix an orthonormal basis $x_{1}^{0}, x_{2}^{0}$ in $C_{2}$ and assume $\left(x_{1}^{\prime}, x_{2}^{\prime}\right)=\left(x_{1}^{0}, x_{2}^{0}\right) B$. Then $\left(x_{1}^{\prime}, x_{2}^{\prime}\right)$ are determined uniquely by $C_{2}$ and $B$. Moreover, $\left[x_{1}^{\prime}, x_{2}^{\prime}\right]$ correspond to $[B]$, and $[B] \in C P^{3} \backslash \bar{M}$, where $\bar{M}=\{[A]: A$ are complex $2 \times 2$ matrices, $\operatorname{det}(A)=0\} . C_{2}$ is associated to a point of Grassmannian manifold $G(3,2)$ and $D_{2}$ is associated to a point of Grassmannian manifold $G(4,2)$, i.e. $x$ is associated to a point of $G(3,2) \times\left(C P^{3} \backslash \bar{M}\right) \times G(4,2)$.

Furthermore, for arbitrary point in $G(3,2) \times\left(C P^{3} \backslash \bar{M}\right) \times G(4,2)$, we can find correspondingly a unique point in $D_{2}^{1}(\mathcal{H})$, and vice versa. In this case, the imbedding is

$$
\begin{aligned}
& f: G(3,2) \times\left(C P^{3} \backslash \bar{M}\right) \times G(4,2) \longrightarrow C P^{11} \\
& p \quad \longrightarrow q
\end{aligned}
$$

where $q=f(p)=x$ and the homogeneous coordinates of $q$ are assumed to be $q=$ $\left(d_{11}, d_{12}, d_{13}, d_{14}, d_{21}, d_{22}, d_{23}, d_{24}, d_{31}, d_{32}, d_{33}, d_{34}\right)$, where $d_{11}=1, d_{12}=a_{12}, d_{13}=a_{12} y_{23}+$ $y_{13}, d_{14}=a_{12} y_{24}+y_{14}, d_{21}=a_{21}, d_{22}=a_{22}, d_{23}=a_{21} y_{13}+a_{22} y_{23}, d_{24}=a_{21} y_{14}+a_{22} y_{24}, d_{31}=$ $x_{13}+a_{21} x_{23}, d_{32}=a_{12} x_{13}+a_{22} x_{23}, d_{33}=y_{23}\left(a_{12} x_{13}+a_{22} x_{23}\right)+y_{13}\left(x_{13}+a_{21} x_{23}\right), d_{34}=$ $y_{24}\left(a_{12} x_{13}+a_{22} x_{23}\right)+y_{14}\left(x_{13}+a_{21} x_{23}\right)$.

The first example tests the theorem from the Segre imbedding point of view. In this case the second factor of the product manifold generates a point. The second one is a lower dimension case according to the Theorem 1.

We have investigated the complex manifold structure for bipartite pure states and the Kähler metric of $D_{k}^{1}(\mathcal{H})$, by presenting explicitly the manifolds with respect to all pure states 
of arbitrarily given Schmidt ranks or Schmidt coefficients and calculating the dimensions of the corresponding manifolds. In fact, we also can express the Kähler metric of $D_{k}^{1}(\mathcal{H})$ by local coordinates, but the expressions are very complicated and it is difficult to compute the geometrical objects such as holomorphic curvature, scalar curvature. It would be also nice to describe the entanglement of quantum states according to some functions of metric or geometrical objects. The results in this paper can be used to study the differential geometry of bipartite mixed states.

Acknowledgment We would like to express gratitude to K. Wu for many helpful suggestions and X.C. Rong, Z.F. Yang for valuable comments. The work is partly supported by NSFC projects 10371014, 10675086, Funds of China Scholarship Council, Funds of Beijing YouXiuRenCai and KM200510028022, NKBRPC(2004CB318000).

[1] M.A. Nielsen and I.L. Chuang, Quantum Computation and Quantum Information, Cambridge University Press, Cambridge, 2000.

[2] C.H. Bennett, D.P. DiVincenzo, J.A. Smolin, and W.K. Wootters, Phys. Rev. A 54, 3824 (1996).

M. Horodecki, Quant. Inf. Comp. 1, 3 (2001).

D. Bruß, J. Math. Phys. 43, 4237 (2002).

W.K. Wootters, Phys. Rev. Lett. 80, 2245(1998).

B.M. Terhal and K.G.H. Vollbrecht, Phys. Rev. Lett. 85, 2625 (2000).

S.M. Fei and X.Q. Li-Jost, Rep. Math. Phys. 53, 195 (2004).

K. Chen, S. Albeverio, and S.M. Fei, Phys. Rev. Lett. 95, 040504 (2005).

K. Chen, S. Albeverio, and S.M. Fei, Phys. Rev. Lett. 95, 210501 (2005). S.M. Fei, Z.X. Wang and H. Zhao, Phys. Lett. A 329(2004)414-419.

[3] M. Lewenstein, D. Bruß, J. I. Cirac, B. Kraus, M. Kuś, J. Samsonowicz, A. Sanpera, and R. Tarrach, J. Mod. Phys. 47, 2481 (2000).

R. Werner, Phys. Rev. A40, 4277 (1989).

A. Peres Phys. Rev. Lett. 77, 1413 (1996).

M. Horodecki, P. Horodecki, and R. Horodecki, Phys. Lett. A 223, 8 (1996).

W. Dür, G. Vidal and J.I. Cirac, Phys. Rev. A 62, 062314(2000). 
S. Karnas and M. Lewenstein, Phys. Rev. A 64, 042313 (2001).

S. Albeverio, S.M. Fei and D. Goswami, Phys. Lett. A 286 (2001)91-96.

S.M. Fei, X.H. Gao, X.H. Wang, Z.X. Wang and K. Wu, Phys. Lett. A 300 (2002)559-566;

Phys. Rev. A 68 (2003) 022315.

S. Albeverio, K. Chen and S.M. Fei, Phys. Rev. A 68(2003)062313.

[4] E.M. Rains, IEEE Transactions on Information Theory 46 54-59(2000).

M. Grassl, M. Rötteler and T. Beth, Phys. Rev. A 58, 1833(1998).

Y. Makhlin, Quant. Info. Proc. 1, 243-252 (2002).

N. Linden, S. Popescu and A. Sudbery, Phys. Rev. Lett. 83, 243 (1999).

S. Albeverio, S.M. Fei, P. Parashar and W.L. Yang, Phys. Rev. A 68 (Rapid Comm.) (2003) 010303.

S. Albeverio, L. Cattaneo, S.M. Fei and X.H. Wang, Int. J. Quant. Inform. 3(2005)603-609; Rep. Math. Phys. 56 (2005)341-350.

S.M. Fei and N.H. Jing, Phys. Lett. A 342(2005)77-81.

C. H. Bennett, S. Popescu, D. Rohrlich, J. A. Smolin, A. V. Thapliyal, Phys. Rev. A 63 (2001) 012307.

W. Dür, G. Vidal, J. I. Cirac, Phys. Rev. A 62, 062314(2000).

F. Verstraete, J.Dehaene, B.De Moor and H. Verschelde Phys. Rev. A. 65, 052112 (2002).

[5] Janusz Grabowski, Giuseppe Marmo, Maret Kus, Geometry of quantum systems:density states and entanglement, J. phys. A 38(2005)10217-10244.

[6] V. I. Man'ko, G. Marmo, E. C. G. Sudarshan, F. Zaccaria, Differential geometry of density states, Rept. Math. Phys. 55(2005)405-422.

[7] J. Grabowski, M. Kuś and G. Marmo, Open Sys. and Information Dyn. 13(2006)343-362. 